\title{
Exploring the cause of conduction delays in patients with repaired Tetralogy of Fallot
}

Citation for published version (APA):

Verzaal, N. J., Masse, S., Downar, E., Nanthakumar, K., Delhaas, T., \& Prinzen, F. W. (2021). Exploring the cause of conduction delays in patients with repaired Tetralogy of Fallot. EP Europace, 23, I105-I112. https://doi.org/10.1093/europace/euaa400

Document status and date:

Published: 01/03/2021

DOI:

10.1093/europace/euaa400

Document Version:

Publisher's PDF, also known as Version of record

Document license:

Taverne

Please check the document version of this publication:

- A submitted manuscript is the version of the article upon submission and before peer-review. There can be important differences between the submitted version and the official published version of record.

People interested in the research are advised to contact the author for the final version of the publication, or visit the DOI to the publisher's website.

- The final author version and the galley proof are versions of the publication after peer review.

- The final published version features the final layout of the paper including the volume, issue and page numbers.

Link to publication

\footnotetext{
General rights rights.

- You may freely distribute the URL identifying the publication in the public portal. please follow below link for the End User Agreement:

www.umlib.nl/taverne-license

Take down policy

If you believe that this document breaches copyright please contact us at:

repository@maastrichtuniversity.nl

providing details and we will investigate your claim.
}

Copyright and moral rights for the publications made accessible in the public portal are retained by the authors and/or other copyright owners and it is a condition of accessing publications that users recognise and abide by the legal requirements associated with these

- Users may download and print one copy of any publication from the public portal for the purpose of private study or research.

- You may not further distribute the material or use it for any profit-making activity or commercial gain

If the publication is distributed under the terms of Article $25 \mathrm{fa}$ of the Dutch Copyright Act, indicated by the "Taverne" license above, 


\title{
Exploring the cause of conduction delays in patients with repaired Tetralogy of Fallot
}

\author{
Nienke J. Verzaal ${ }^{1}$, Stéphane Massé2 ${ }^{2}$ Eugene Downar ${ }^{2}$, \\ Kumaraswamy Nanthakumar ${ }^{2}$, Tammo Delhaas $\mathbb{1}^{3}{ }^{3}$, and Frits W. Prinzen ${ }^{1} *$
}

\begin{abstract}
${ }^{1}$ Department of Physiology, Maastricht University, PO Box 616, 6200 MD Maastricht, the Netherlands; ${ }^{2}$ The Hull Family Cardiac Fibrillation Management Laboratory, Toronto General Hospital, University Health Network, 200 Elizabeth Street, Toronto, Ontario M5G 2C4, Canada; and ${ }^{3}$ Department of Biomedical Engineering, Maastricht University, PO Box 616, 6200 MD Maastricht, the Netherlands
\end{abstract}

Received 27 November 2020; editorial decision 2 December 2020; accepted after revision 4 December 2020

Aims

Cardiac dyssynchrony in patients with repaired Tetralogy of Fallot ( $r$ ToF) has been attributed to right bundle branch block (RBBB), fibrosis and/or the patches that are inserted during repair surgery. We aimed to investigate the basis of abnormal activation in $\mathrm{rToF}$ patients by mapping the electrical activation sequence during sinus rhythm (SR) and right ventricular (RV) pacing.

Methods A total of 17 patients were studied [13 with rToF, 2 with left bundle branch block (LBBB), and 2 without RBBB or and Results LBBB (non-BBB)] during medically indicated cardiac surgery. During SR and RV pacing, measurements were performed using 112-electrode RV endocardial balloons (rToF only) and biventricular epicardial sock arrays (four of the rToF and all non-rToF patients). During SR, functional lines of block occurred in five rToF patients, while RV pacing caused functional blocks in four rToF patients. The line of block persisted during both SR and RV pacing in only 2 out of 13 rToF patients. Compared to SR, RV pacing increased dispersion of septal activation, but not dispersion of endocardial and epicardial activation of the RV free wall. During pacing, RV and left ventricular activation dispersion in rToF patients were comparable to that of the non-rToF patients.

Conclusion The results of the present study indicate that the delayed activation in the right ventricle of rToF patients is predominantly due to block(s) in the Purkinje system and that conduction in RV tissue is fairly normal.

Keywords Repaired Tetralogy of Fallot • Pacing • Mapping • Dyssynchrony • Congenital heart disease • Conduction abnormalities

\section{Introduction}

Tetralogy of Fallot (ToF), the most common congenital cyanotic cardiac disease, is characterized by a ventricular septal defect (VSD), overriding aorta, pulmonary stenosis, and concomitant hypertrophy of the right ventricle. ${ }^{1}$ During surgery, which is usually performed at a very young age, the VSD is closed and the pulmonary stenosis is alleviated. While surgery has a high chance of success to restore anatomy, it often results in right bundle branch block (RBBB), post-operative pulmonary insufficiency and residual stenosis. These three conditions are potentially harmful for proper cardiac function and may negatively affect the prognosis of patients with repaired Tetralogy of Fallot (rToF). Patients with rToF often display dyssynchrony, and larger dyssynchrony is correlated to lower exercise capacity and increased risk of ventricular arrhythmias. ${ }^{2}$

An obvious candidate cause of this dyssynchrony is damage to part of the rapid conduction system, in particular the right bundle branch. ${ }^{3}$ Electrophysiological studies performed during rToF surgery showed $\mathrm{RBBB}^{3,4}$ and also left anterior hemiblock has been found post-surgery. ${ }^{5}$

However, structural abnormalities (like fibrosis) due to longer lasting right ventricular (RV) overload and related RV dilation, surgical scar and the patch used to relieve pulmonary stenosis may cause or aggravate the dyssynchrony. ${ }^{6,7}$

The present study aims to assess if, and to what extent, abnormal activation in rToF hearts is due to structural tissue abnormalities, by examining the presence of conduction block and dispersion of 


\section{What's new?}

- Electrical dyssynchrony in hearts of patients with Tetralogy of Fallot late after surgical repair (rToF) may be caused by damage to the rapid conduction system or by disturbed conduction in the working myocardium.

- We found that in rToF patients structural conduction block is rare and that conduction properties of the working myocardium are comparable to those in non-rToF patients.

- These findings support the idea that cardiac resynchronization therapy may be a valuable adjunct therapy in rToF patients.

activation using detailed endo- and epicardial mapping. Measurements during sinus rhythm (SR) and RV pacing allowed us to distinguish between the conductive effects of the His-Purkinje system and myocardial tissue properties and also between structural and functional blocks. Data from rToF patients was compared with a small group of non-rToF patients.

\section{Methods}

\section{Study population and mapping array}

Studies were performed in Toronto General Hospital, between 1995 and 2008. This study was approved by the University Health Network Ethics Review Board. All patients provided informed consent prior to inclusion.

During medically indicated cardiac surgery (redo pulmonary valve replacement), detailed RV intraoperative mapping was performed for ventricular tachycardia in $13 \mathrm{rToF}$ patients [ 4 women, 9 men, age $37 \pm 8$ [mean \pm standard deviation (SD)]; 8 with left ventricular (LV) ejection fraction $>60 \%$, 5 with mild to moderate LV dysfunction (LV ejection fraction $<60 \%)$ ]. An RV cavity-shaped endocardial balloon array was used that consisted of 112 bipolar electrodes (Figure 1A). Additionally, in four of these patients, an epicardial sock array with 112 bipolar electrodes (Figure $1 B$ ) was used to map both LV and RV activation. The latter array was also used in two patients with left bundle branch block (LBBB) (one woman, one man, age 61) and two patients without either RBBB or LBBB (non-BBB) (both men, age 64).

The arrays and data on total activation times and regions of late activation during SR have been described previously. ${ }^{8,9}$ For the present study, we selected patients in whom also mapping data were available during RV pacing. Briefly, the 112 electrode pairs on each of the arrays were divided over 14 vertical splines of 8 pairs each. For all 112 channels, unipolar (sampling rate $1000 \mathrm{~Hz}$, filtering $0.5-200 \mathrm{~Hz}$ ) and bipolar signals (sampling rate $2000 \mathrm{~Hz}$, filtering $28-750 \mathrm{~Hz}$ ) were recorded simultaneously. The electrodes were assigned a region (details in legends to Figures 2 and 3).

In the four patients where both arrays were used, the sites of earliest activation on both sock and balloon were overlaid during analysis of paced maps, to ensure proper assignment of the electrodes on the sock to either left ventricle or right ventricle on the epicardium.

In order to increase the number of observations in LBBB hearts, relevant data from literature describing four patients who underwent electrocardiographic imaging measurements during both SR and RV pacing were included. ${ }^{10}$

\section{Data acquisition and analysis}

Mapping was performed after the initiation of cardiopulmonary bypass. Unipolar and bipolar electrograms were recorded, using an epicardial needle stitched onto the base of the heart as a reference. Measurements were performed during SR and during RV pacing from an epicardial site.

Unipolar and bipolar electrograms were analysed using customwritten software to determine local activation times, using the onset of the QRS complex or the middle of the pacing spike as time reference.
A

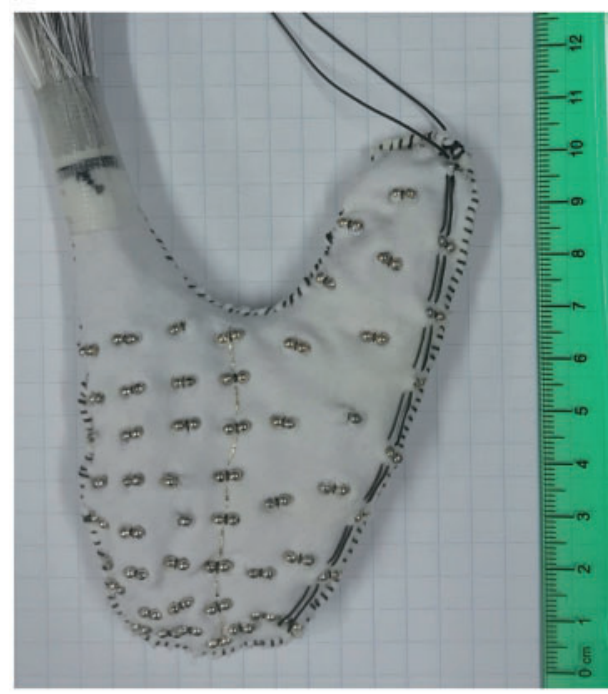

B

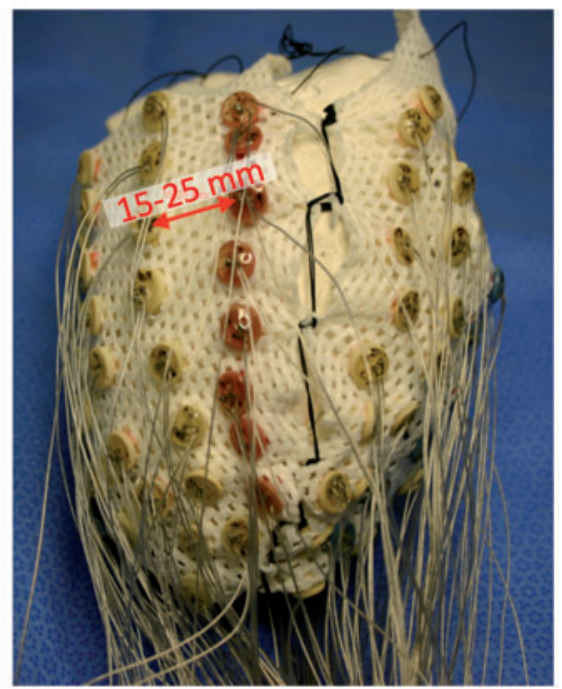

Figure I (A) The endocardial right ventricular (RV) balloon array. The thumb-like structure on the right side was positioned inside the RV outflow tract. (B) The epicardial sock array. 


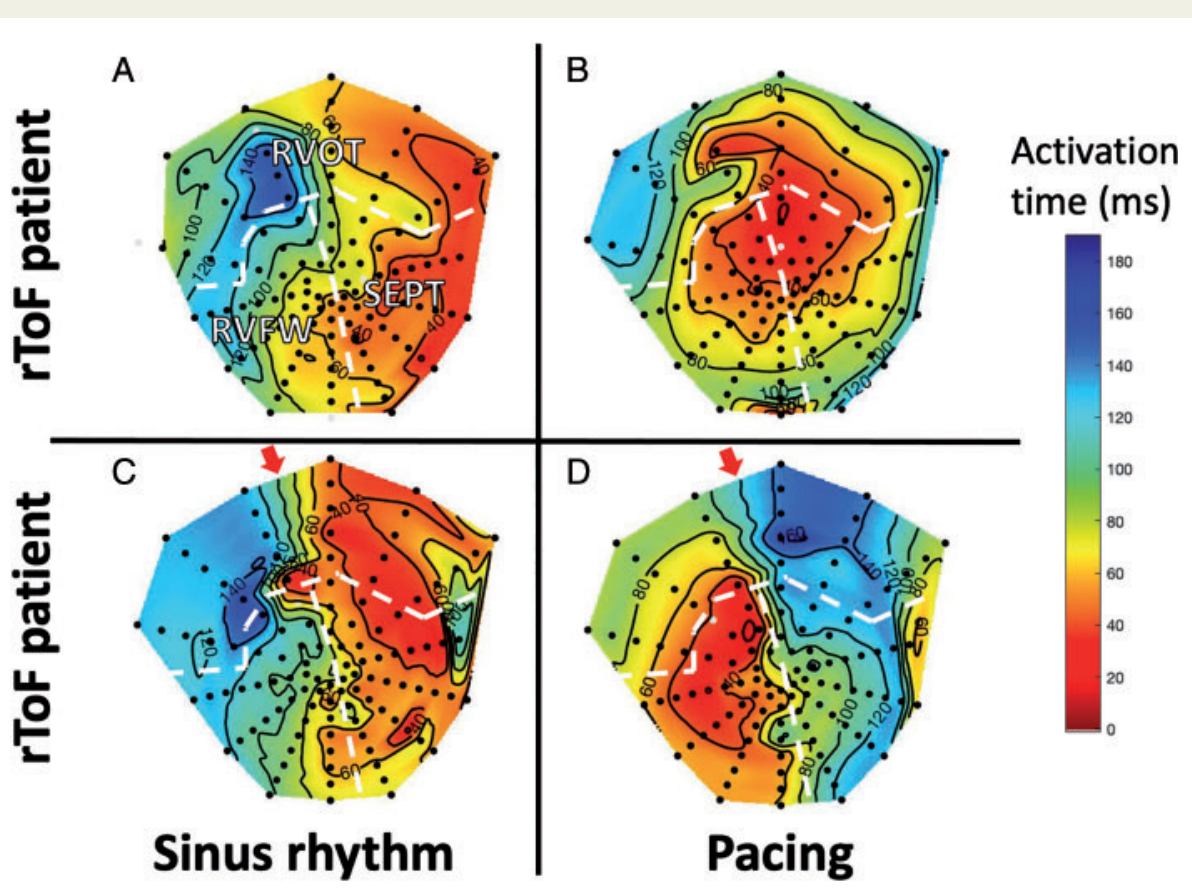

Figure 2 Activation maps of the right ventricular (RV) balloon for two patients with repaired Tetralogy of Fallot (rToF), during sinus rhythm (A, $C$ ) and pacing $(B, D)$. The white lines indicate the borders between the different areas: RV free wall (RVFW), septum (SEPT), and RV outflow tract (RVOT). The red arrows point to the structural line of block. Grey dots denote electrodes with poor signal quality, omitted from analysis.

Using custom-written MATLAB software, activation patterns were visualized in activation maps, and several parameters were calculated:

- Average activation time (ms) per region: allowing gross comparison of the order of activation between regions.

- Regional activation dispersion (ms): the difference between the 5\% and $95 \%$ percentile of activation times in each region.

- Total activation time (ms): the latest activation time of the epicardial sock array.

\section{Statistical analysis}

Statistical analysis was performed using GraphPad Prism 8 (GraphPad Software, San Diego, USA). Differences in activation times and regional activation dispersions per region of the balloon were assessed using a Friedman test, followed by a Dunn's multiple comparison test. Activation dispersions of the whole balloon during SR and pacing were compared using a Wilcoxon matched pairs signed rank test. A $P$-value $\leq 0.05$ was considered to be significant. Due to the small number of patients who were examined using the sock array, no statistical analysis was performed for this data.

\section{Results}

\section{Endo- and epicardial activation maps}

For the majority of the rToF patients, endocardial RV activation (measured on the balloon array) started on the septum and then spread to the RV free wall (RVFW) and RV outflow tract (RVOT) (Figure 2A and C). Upon RV pacing, the RVFW became activated earlier than the septum and the RVOT (Figure 2B and D).
Both during SR and during pacing functional blocks occurred. The locations of these blocks varied considerably between patients and between activation modes. In seven rToF patients, activation progressed fairly regularly during SR, while five patients had a functional block that disappeared during pacing (example of the latter in Figure $2 A$ and $B)$. These functional blocks occurred in the RVOT $(n=3)$, septum $(n=2)$, RVFW $(n=1)$, or in the septum/RVOT border area $(n=1)$. (Note that a patient can have more than one block.)

In the remaining patient and in one of the patients with functional block, a structural block was present (example in Figure 2C and D). During pacing, functional blocks appeared in four patients, in the RVOT $(n=3)$, septum $(n=1)$ and in the septum/RVFW border area $(n=1)$. During SR, no blocks were present in two out of these four patients.

During SR, the RVFW was the main late activated area in two of the four rToF patients mapped epicardially (example in Figure 3A, paced map in Figure 3B), whereas in one patient this was the anterobasal area of both right ventricle and left ventricle (Figure $3 C$ ) and in another patient the left ventricle.

For the non-BBB patients, during SR the latest activated area was found in the left ventricle (one patient, Figure $4 A$ ) or the right ventricle (one patient), while in both LBBB patients, the latest activation occurred in the left ventricle (Figure 4C).

In all patient groups, RV pacing created early activation near the pacing electrode and spread of activation towards the opposite wall.

No epicardial lines of block were observed during SR in any of the four rToF patients, whereas during RV pacing, a line of block occurred in the left ventricle in one patient (Figure 3D). In the non-BBB patients, no lines of block were observed during either SR or RV 


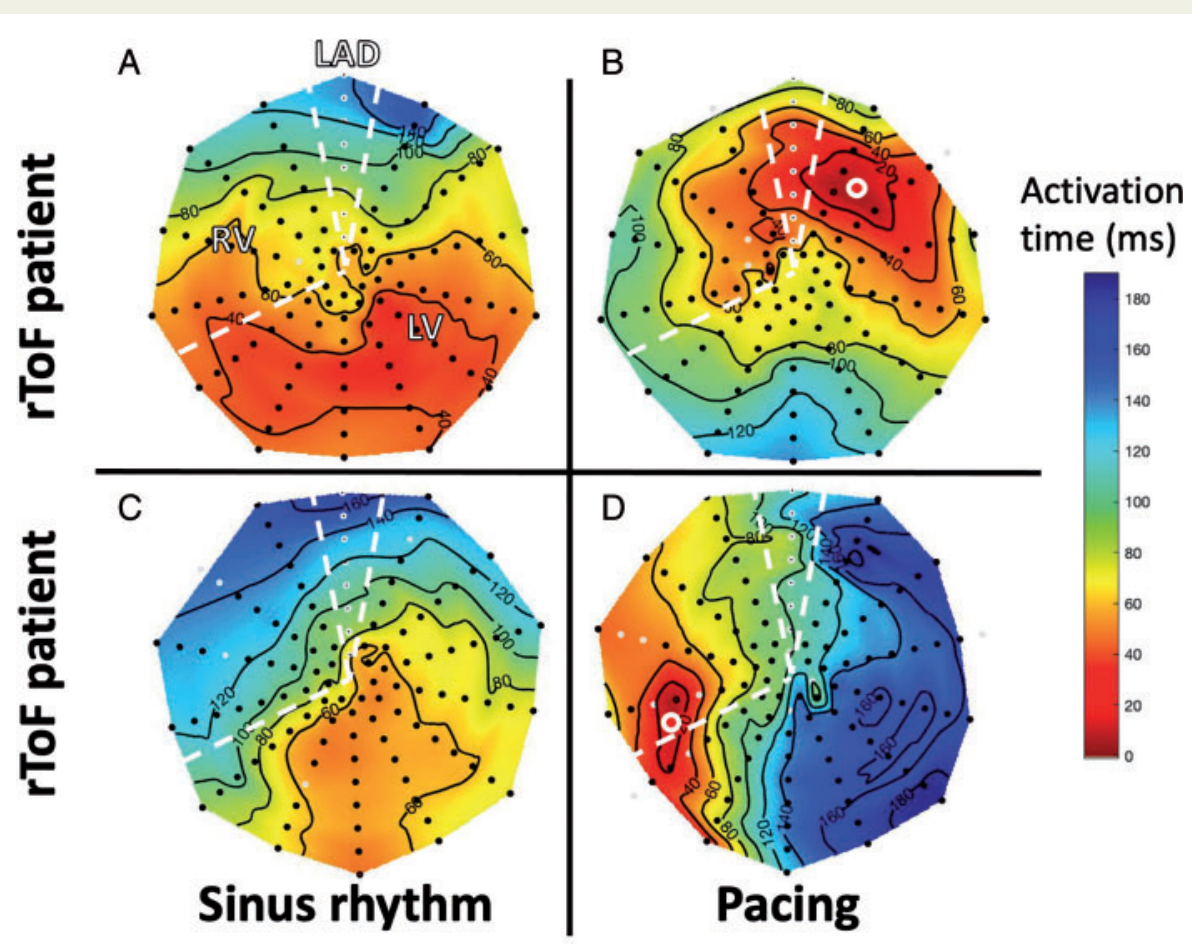

Figure 3 Activation maps of the epicardial sock for two patients with repaired Tetralogy of Fallot (rToF), during sinus rhythm ( $A, C$ and pacing ( $B$, $D)$. One spline is aligned with the left anterior descending artery (LAD), the white lines indicate the borders between the different areas: left ventricle $(\mathrm{LV})$, right ventricle $(\mathrm{RV})$, and LAD. The white circles indicate the pacing sites in the right panels. Grey dots denote electrodes with poor signal quality, omitted from analysis.

pacing. In one of the LBBB patients, a persistent line of block occurred in the left ventricle (Figure $4 C$ and D), while in the other LBBB patient, a line of block was observed during LV pacing.

In summary, functional blocks, that (dis)appeared during ventricular pacing, were found in 8 out of 13 rToF patients, while only 2 had a structural conduction block. One of the two LBBB patients had a functional block and the other patient had a structural block.

\section{Quantification of cardiac activation patterns}

Data from all $r$ ToF patients show that during SR the RVFW $(92 \pm 10 \mathrm{~ms}$, mean $\pm \mathrm{SD}, P=0.001)$ and RVOT $(85 \pm 19 \mathrm{~ms}, P=0.068)$ are later activated than the septum $(57 \pm 11 \mathrm{~ms})$, whereas RV pacing results in earlier activation of the RVFW $(57 \pm 9 \mathrm{~ms} ; P=0.001 \mathrm{vs}$. SR $)$ and later activation of the septum $(94 \pm 18 \mathrm{~ms} ; P<0.001 \mathrm{vs}$. RVFW) and RVOT $(114 \pm 22 \mathrm{~ms} ; P<0.0001$, Figure $5 \mathrm{~A})$.

Average epicardial LV activation time of rToF patients [67 (55-83) $\mathrm{ms}$, mean and range] and non-BBB patients (51 and $77 \mathrm{~ms}$ ) was comparable, whereas it was larger in the LBBB patients [114 (88-144) $\mathrm{ms}$ ] than in all rToF and non-BBB patients (Figure 5B). Pacing increased the average epicardial LV activation time for all but one patient [rToF 101 (73-132) ms; non-BBB 98 and 183 ms; LBBB 133 (121-149) ms]. Mean increase in rToF was $34 \mathrm{~ms}, 77 \mathrm{~ms}$ in non-BBB and $19 \mathrm{~ms}$ in LBBB (Figure 5B). On average, mean RV activation time was larger in rToF patients [95 (73-117) ms] than in the non-BBB (21 and $113 \mathrm{~ms}$ ) and the LBBB patients [46 (29-84) ms; Figure 5C]. During pacing, average RV activation occurred consistently earlier in the $r$ ToF patients while it became later in the other patients [rToF 65 (53-71) ms, non-BBB 61 and $150 \mathrm{~ms}$, LBBB 85 (36-188) ms]. The mean change in activation times was $-30 \mathrm{~ms}$ (rToF), $+39 \mathrm{~ms}$ (non$\mathrm{BBB}$ ) and $+38 \mathrm{~ms}$ (LBBB); Figure 5C.

Total epicardial activation times were comparable between rToF patients [161 ms (151-170) mean and range], LBBB patients (174 ms for both), and one of the non-BBB patients (95 and $150 \mathrm{~ms}$ ) during $S R$. During ventricular pacing, total activation time increased in the non-BBB patients (to 146 and $242 \mathrm{~ms}$ ) and LBBB patients (to 220 and $224 \mathrm{~ms}$ ), whereas it did not consistently change in the rToF patients [161 ms (133-188)].

\section{Activation dispersion}

The rToF patients had a total activation dispersion within the RV balloon of $93 \mathrm{~ms}$ (range 49-114 ms) during SR. Activation dispersion did not differ between septum $(57 \pm 27 \mathrm{~ms})$ and RVFW during SR (61 $\pm 15 \mathrm{~ms}$ ), but during pacing, septal activation dispersion increased significantly (to $78 \pm 6 \mathrm{~ms} ; P=0.038$ vs. septum $S R$ and $P=0.027$ vs. RVFW pacing; Figure 5D). This increase is likely to be largely responsible for the significant increase in total activation dispersion during RV pacing [to $114 \mathrm{~ms}(83-141 \mathrm{~ms}), P=0.006$ ].

During SR, epicardial LV activation dispersion was smallest in the non-BBB patients ( 35 and $62 \mathrm{~ms}$ ), intermediate in three out of four rToF patients [82 (64-128) ms, mean and range] and largest in the LBBB patients (110 and $132 \mathrm{~ms}$; Figure 5E). During RV pacing, LV 


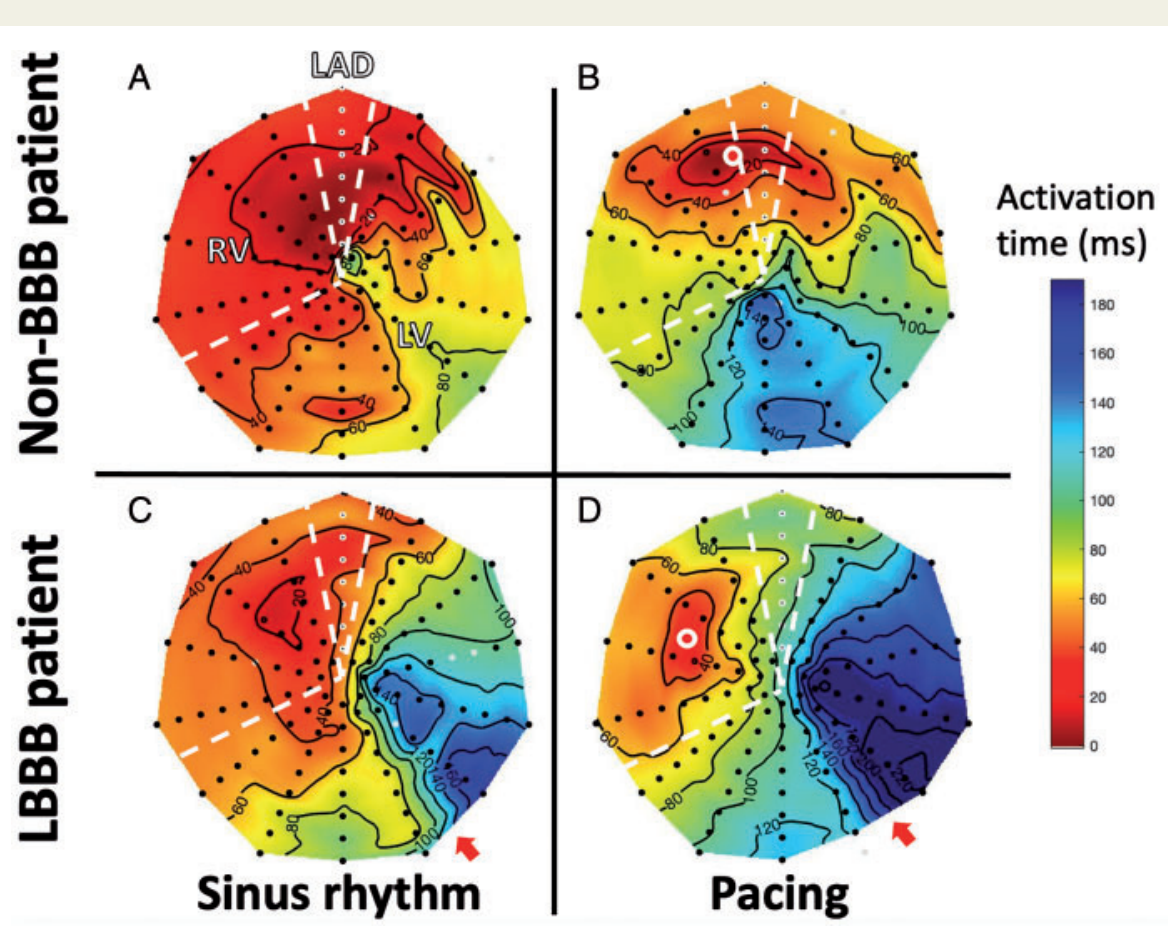

Figure 4 Activation maps of the epicardial sock for one patient without either right or left bundle branch block (non-BBB) (A and $B)$ and one patient with left bundle branch block ( $L B B B)(C$ and $D)$ during sinus rhythm $(A, C)$ and pacing $(B, D)$. The red arrows point to the structural line of block. The white circles indicate the pacing sites in the right panels. Grey dots denote electrodes with poor signal quality, omitted from analysis. Area abbreviations as in Figure 3.

activation dispersion increased in all but one rToF and one LBBB patient so that activation dispersion became comparable between the three patient groups [rToF 103 (65-125) ms, non-BBB 92 and $131 \mathrm{~ms}$, LBBB 121 and $140 \mathrm{~ms}$; Figure 5E].

On average, epicardial RV activation dispersion was larger in the $r$ ToF patients [74 (63-101) ms] than in the other patients (non-BBB 26 and $62 \mathrm{~ms}$, LBBB 31 and $65 \mathrm{~ms}$; Figure 5F). This dispersion decreased upon RV pacing in two of the four rToF patients, while increasing in the non-BBB patients and one LBBB patient. Importantly, this made RV dispersion of activation comparable between the patient groups [rToF 59 (54-66) ms; non-BBB 70 and 132 ms, LBBB 57 and $58 \mathrm{~ms}$ ] and one non-BBB patient showed the largest activation dispersion (Figure 5F).

\section{Discussion}

The main findings of the present study are that in rToF patients (i) structural lines of block in the right ventricle are rare; (ii) dispersion of activation in both endo- and epicardium of the RVFW is virtually unchanged between SR and RV pacing; and (iii) during RV pacing, epicardial LV and RV activation dispersion in rToF patients is comparable with that in non-rToF patients. These findings indicate that conduction properties of the working myocardium are fairly well-preserved and that the delayed activation in the right ventricle of $\mathrm{rToF}$ patients is likely in large part due to blocks in the Purkinje system. The latter has important implications for the potential application of cardiac resynchronization therapy (CRT) in these patients. The persistence of lines of block in only two out of the 13 patients indicates that structural regions of block were present in only a minority of $r$ ToF patients. Functional blocks during SR were present in about $40 \%$ of the patients, and during pacing in approximately $30 \%$.

\section{Lines of block in rToF patients}

In the majority of rToF patients (11/13), there were no lines of structural block that persisted during both SR and pacing. We consider this finding to be evidence for the idea that a defect of (part of) the rapid conduction system, in particular the right bundle branch, is the primary cause of the abnormal conduction in rToF hearts. Indeed, RBBB morphology of the QRS complex is a common finding after surgical repair of ToF, 6,11 which has been attributed to damage to the right bundle branch due to VSD closure, RV ventriculotomy or resection of stenotic tissue. . $^{3,4}$

The evidence of lines of functional block may, however, point to some conduction problems at the tissue level, such as fibrosis and electrical remodelling. In 8 out of the 13 rToF patients, functional lines of block were visible endocardially during SR and/or pacing. The (dis)appearance of lines of block may be explained by the fact that collagen can affect conduction in an anisotropic manner, meaning that activation of the tissue in one direction will lead to conduction block, while conduction in another direction is not negatively influenced. ${ }^{7,12}$ Since the functional blocks often occurred in the RVOT (in 5/8 patients who had functional block during either SR or pacing), we may speculate that surgical scars are (partially) responsible for these blocks. 

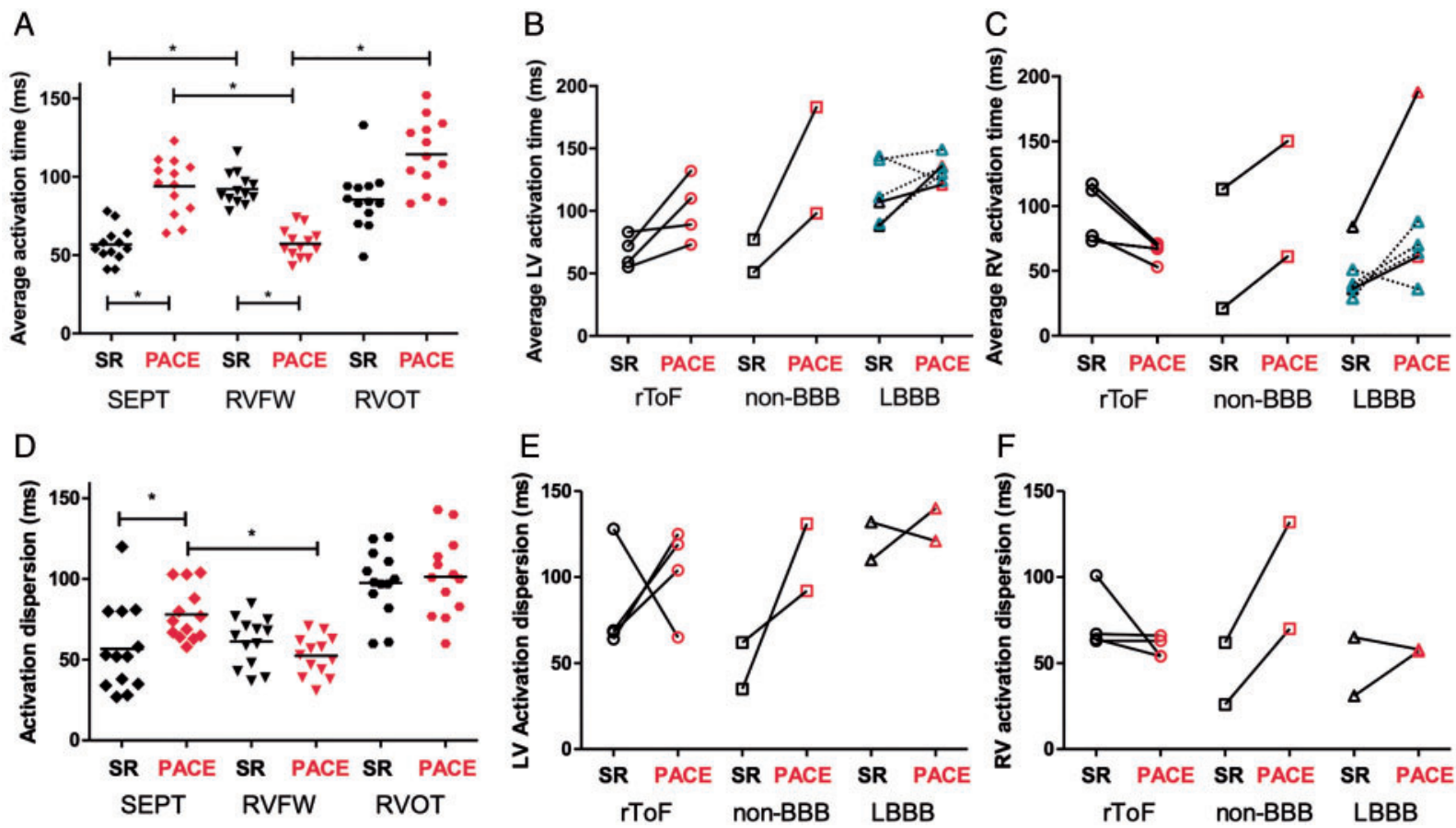

Figure 5 Activation times in different zones of the endocardial RV balloon array $(A)$ and at the LV $(B)$ and RV epicardium (C). Dispersion of activation times in different zones of the endocardial RV balloon array $(D)$ and at the $L V(E)$ and RV epicardium $(F)$. Black symbols denote sinus rhythm (SR), red symbols denote pacing (PACE), other abbreviations as in Figures 2-4. Turquoise triangles in $B$ and $C$ denote data obtained from a study using electrocardiographic imaging. ${ }^{10}$ Horizontal lines in panels $A$ and $D$ represent the mean values. $* P \leq 0.05$ between the indicated values.

Additionally, since fibrotic tissue has been found outside of the patches in RVOT and VSD both in rToF patients ${ }^{13}$ and in animal models of $r$ ToF, ${ }^{14,15}$ fibrosis may also have played a role in functional blocks outside of the RVOT. However, delays in regions with fibrosis are also governed by the length of fibrotic strands, with short strands hardly affecting conduction. ${ }^{7}$ It is therefore possible that fibrotic tissue did not automatically cause conduction block, which may be an explanation for the relative rareness of epicardial functional conduction blocks in rToF patients (one out of four).

Another potential cause of lines of functional block is electrical remodelling and one protein that may be involved in this process is connexin 43 (Cx43). Its expression can decrease, and it may be found in a more lateral location on cardiomyocytes. Cx43 abnormalities have been shown in an animal model of rToF, ${ }^{14,15}$ and also in RV samples of rToF patients. ${ }^{16}$

Unfortunately, no imaging data regarding cardiac fibrosis was available in any of the patients, but we can speculate about the reasons for finding structural blocks in the RVOT, particularly at the junction of the RVFW and the septum. Firstly, fibrotic tissue has been frequently observed at the RVFW-septum junction in patients with rToF. ${ }^{17}$ Secondly, even in normal hearts tissue architecture is less organized at the ventricular junctions than elsewhere in the ventricular walls. ${ }^{18}$ It should be noted, however, that the patients that were included in the present study all underwent electrical mapping to search for foci of arrhythmia, meaning that they represent the patients with the most severely affected tissue properties in their respective categories.

\section{Activation dispersion in rToF during ventricular pacing}

In this study, we used ventricular pacing to specifically test conduction properties in the working myocardium. Doing so, it was observed that epicardial activation dispersion in both right ventricle and left ventricle of rToF patients was quite comparable to that in nonrToF patients. Therefore, these data indicate that even if there are demonstrable tissue conduction abnormalities (structural and/or functional block), overall conduction is hardly affected.

During RV pacing, endocardial RV activation dispersion did not increase in the RVOT or RVFW. This further supports the notion that cardiac tissue properties of the majority of the RV areas are similar, since conditions like extensive fibrosis, electrical uncoupling or patch are expected to create zones of conduction delay or blocks. ${ }^{6,7,12}$ The fact that comparable values of RVFW activation dispersion were measured using endocardial and epicardial mapping and that endocardially and epicardially measured dispersion changed comparably between SR and pacing further underlines the relative tissue homogeneity in rToF hearts.

\section{Extrapolation to resynchronization of rToF patients}

Since RBBB in rToF patients is so prevalent, RV resynchronization through CRT is a tantalizing possibility. This may be especially true in hearts with RV hypertrophy (which may remain present in patients with rToF, due to residual pulmonary stenosis), since studies in dogs 
have shown that the negative haemodynamic effects of RBBB are more pronounced if the right ventricle is hypertrophied. ${ }^{19}$ Moreover, dyssynchrony itself leads to extensive ventricular remodelling, potentially further worsening ventricular function and increasing the risk of arrhythmia. $^{20}$

Analogous to the abnormal contraction pattern observed in the left ventricle of patients with $\mathrm{LBBB}$, patients with $r$ ToF and RBBB show dyssynchronous contraction in the right ventricle. ${ }^{21}$ Indeed, several small studies have shown that CRT can improve synchrony of activation and cardiac function in patients with rToF, either through RV pacing aimed at fusion with the intrinsic activation wave front or through biventricular pacing. ${ }^{22-24}$ Even more interesting is the finding in a combined patient and computer simulation study that correction of the dyssynchrony in rToF patients may be of larger benefit to RV function and exercise tolerance than surgical correction of pulmonary valve regurgitation. ${ }^{25}$

Although we did not investigate CRT, our findings underline the potential to resynchronize the ventricles because our data primarily indicate conduction blocks proximal in the rapid conduction system. Moreover, because functional lines of block are also commonly found in patients with $\mathrm{LBBB}^{26}$ (in whom CRT has become an established treatment ${ }^{27}$ ) we do not believe that their presence precludes successful CRT in rToF patients. In addition, activation dispersion in the right ventricle and left ventricle during ventricular pacing was comparable in $\mathrm{rToF}$ and in LBBB patients.

\section{Limitations}

This study has several limitations. Firstly, the number of patients that was studied was small and no healthy controls were included. Both are not surprising given the invasive nature of the mapping procedure. Only one pacing site was studied, and this site was not standardized for all patients. In comparison to studies focusing on the presence of isthmuses leading to ventricular arrhythmias, ${ }^{28}$ the density of electrodes in the most basal part of the RVOT of the sock and/ or balloon mapping arrays used in the present study may have been somewhat lower.

\section{Conclusions}

Approximately three decades after repair surgery structural lines of block are rare in rToF patients. Dispersion of activation in both endo- and epicardium of the RVFW is virtually unchanged between SR and RV pacing. Furthermore, activation dispersion in both right ventricle and left ventricle during pacing is comparable with that in non-BBB and LBBB patients. These findings indicate that the delayed activation in the right ventricle of $r$ ToF patients is predominantly due to blocks in the Purkinje system, while conduction properties in the right ventricle are relatively normal.

\section{Funding}

This paper is part of a supplement supported by an unrestricted grant from the Theo-Rossi di Montelera (TRM) foundation.

Conflict of interest: F.W.P. received research grants from Medtronic, Abbott, Microport CRM, Biotronik, Pie Medical
Imaging and EBR Systems. K.N. is a consultant for Servier, Biosense Webster, Abbott and BlueRock Therapeutics. S.M. is a consultant for Abbott.

\section{Data availability}

The data underlying this article cannot be shared publicly due to the privacy of the patients that participated in the study. The data will be shared on reasonable request to the corresponding author.

\section{References}

1. Apitz C, Webb GD, Redington AN. Tetralogy of Fallot. Lancet 2009;374: 1462-71.

2. D'Andrea A, Caso P, Sarubbi B, D'Alto M, Giovanna Russo M, Scherillo M et al. Right ventricular myocardial activation delay in adult patients with right bundle branch block late after repair of Tetralogy of Fallot. Eur J Echocardiogr 2004;5: 123-31.

3. Horowitz LN, Alexander JA, Edmunds LH. Postoperative right bundle branch block: identification of three levels of block. Circulation 1980;62:319-28.

4. Horowitz LN, Simson MB, Spear JF, Josephson ME, Moore EN, Alexander JA et al. The mechanism of apparent right bundle branch block after transatrial repair of Tetralogy of Fallot. Circulation 1979;59:1241-52.

5. Steeg CN, Krongrad E, Davachi F, Bowman FO, Malm JR, Gersony WM. Postoperative left anterior hemiblock and right bundle branch block following repair of Tetralogy of Fallot. Clinical and etiologic considerations. Circulation 1975; 51:1026-9.

6. Jalal Z, Sacher F, Fournier E, Cochet H, Derval N, Haissaguerre M et al. Right Ventricular electrical activation in patients with repaired Tetralogy of Fallots. Circ Arrhythm Electrophysiol 2019;12:e007141.

7. Kawara T, Derksen R, de Groot JR, Coronel R, Tasseron S, Linnenbank AC et al. Activation delay after premature stimulation in chronically diseased human myocardium relates to the architecture of interstitial fibrosis. Circulation 2001;104: 3069-75.

8. Nanthakumar K, Massé S, Poku K, Silversides CK, Chauhan VS, Mariani JA et al. Intraoperative high-density global mapping in adult-repaired Tetralogy of Fallot. Altered left ventricular and right ventricular activation and implications for resynchronization strategies. J Am Coll Cardiol 2010;55:2409-11.

9. Massé S, Downar E, Chauhan V, Sevaptsidis E, Nanthakumar K. Ventricular fibrillation in myopathic human hearts: mechanistic insights from in vivo global endocardial and epicardial mapping. AJP Hear Circ Physiol 2007;292:H2589-97.

10. Jia P, Ramanathan C, Ghanem RN, Ryu K, Varma N, Rudy $Y$ Electrocardiographic imaging of cardiac resynchronization therapy in heart failure: observation of variable electrophysiologic responses. Hear Rhythm 2006;3 296-310.

11. De Ruijter FTH, Weenink I, Hitchcock FJ, Meijboom EJ, Bennink GBWE. Right ventricular dysfunction and pulmonary valve replacement after correction of Tetralogy of Fallot. Ann Thorac Surg 2002;73:1794-800.

12. Dhein S, Seidel T, Salameh A, Jozwiak J, Hagen A, Kostelka M et al. Remodeling of cardiac passive electrical properties and susceptibility to ventricular and atrial arrhythmias. Front Physiol 2014;5:424.

13. Cochet H, Iriart X, Allain-Nicolaï A, Camaioni C, Sridi S, Nivet H et al. Focal scar and diffuse myocardial fibrosis are independent imaging markers in repaired tetralogy of Fallot. Eur Heart J Cardiovasc Imaging 2019;20:990-1003.

14. Benoist D, Dubes V, Roubertie F, Gilbert SH, Charron S, Constantin M et al. Proarrhythmic remodelling of the right ventricle in a porcine model of repaired tetralogy of Fallot. Heart 2017;103:347-54.

15. Dubes V, Benoist D, Roubertie F, Gilbert SH, Constantin M, Charron S et al. Arrhythmogenic remodeling of the left ventricle in a porcine model of repaired Tetralogy of Fallot. Circ Arrhythm Electrophysiol 2018;11:e006059.

16. Kołcz J, Drukała J, Bzowska M, Rajwa B, Korohoda W, Malec E. The expression of connexin 43 in children with Tetralogy of Fallot. Cell Mol Biol Lett 2005;10: 287-303.

17. Babu-Narayan SV, Kilner PJ, Li W, Moon JC, Goktekin O, Davlouros PA et al. Ventricular fibrosis suggested by cardiovascular magnetic resonance in adults with repaired Tetralogy of Fallot and its relationship to adverse markers of clinical outcome. Circulation 2006;113:405-13.

18. Kuribayashi T, Roberts WC. Myocardial disarray at junction of ventricular septum and left and right ventricular free walls in hypertrophic cardiomyopathy. Am J Cardiol 1992;70:1333-40.

19. Yasui $H$, Yoshitoshi M, Komori M, Tominaga R, Kawachi $Y$, Ueno $Y$ et al Cardiodynamic effects of experimental right bundle branch block in canine hearts with normal and hypertrophied right ventricles. Am Heart J 1985;109: 69-77. 
20. Nguyên UC, Verzaal NJ, van Nieuwenhoven FA, Vernooy K, Prinzen FW. Pathobiology of cardiac dyssynchrony and resynchronization therapy. Europace 2018;20:1898-909.

21. Hui W, Slorach C, Dragulescu A, Mertens L, Bijnens B, Friedberg MK. Mechanisms of right ventricular electromechanical dyssynchrony and mechanical ineffciency in children after repair of tetralogy of Fallot. Circ Cardiovasc Imaging 2014;7:610-8.

22. Thambo J-B, De Guillebon M, Xhaet O, Dos Santos P, Roubertie F, Labrousse L et al. Biventricular pacing in patients with Tetralogy of Fallot: non-invasive epicardial mapping and clinical impact. Int J Cardiol 2013;163:170-4.

23. Vojtovič $P$, Kučera F, Kubuš $P$, Gebauer R, Matějka T, Tláskal T et al. Acute right ventricular resynchronization improves haemodynamics in children after surgical repair of tetralogy of Fallot. Europace 2018;20:323-8.

24. Plymen CM, Finlay M, Tsang V, O'leary J, Picaut N, Cullen S et al. Haemodynamic consequences of targeted single- and dual-site right ventricular pacing in adults with congenital heart disease undergoing surgical pulmonary valve replacement. Europace 2015;17:274-80.
25. Lumens J, Fan C-PS, Walmsley J, Yim D, Manlhiot C, Dragulescu A et al. Relative impact of right ventricular electromechanical dyssynchrony versus pulmonary regurgitation on right ventricular dysfunction and exercise intolerance in patients after repair of Tetralogy of Fallot. J Am Heart Assoc 2019;8: e010903.

26. Auricchio A, Fantoni C, Regoli F, Carbucicchio C, Goette A, Geller C et al. Characterization of left ventricular activation in patients with heart failure and left bundle-branch block. Circulation 2004;109:1133-9.

27. Brignole M, Auricchio A, Baron-Esquivias G, Bordachar P, Boriani G, Breithardt O-A et al. 2013 ESC guidelines on cardiac pacing and cardiac resynchronization therapy: the task force on cardiac pacing and resynchronization therapy of the European Society of Cardiology (ESC). Developed in collaboration with the European Heart Rhythm Association. Europace 2013;34:2281-329.

28. Kapel GFL, Sacher F, Dekkers OM, Watanabe M, Blom NA, Thambo J-B et al. Arrhythmogenic anatomical isthmuses identified by electroanatomical mapping are the substrate for ventricular tachycardia in repaired Tetralogy of Fallot. Eur Heart / 2017;38:268-76 